Check for updates

Cite this: Phys. Chem. Chem. Phys., 2019, 21, 6776

Received 25th January 2019, Accepted 5th March 2019

DOI: 10.1039/c9cp00500e

rsc.li/pccp

\section{Previtamin D: Z-E photoisomerization via a Hula-twist conical intersection}

\begin{abstract}
Werner Fuß 1
On photoisomerization of previtamin D - a steroid Z-triene - produced in situ by ring opening of 7-dehydrocholesterol in a cold matrix, it was found in A. M. Müller et al. [Angew. Chem., Int. Ed., 1998, 37, 505-507] that the product (tachysterol) had rotated not only its central double bond but also an adjacent single bond. This is called a Hula twist (HT) due to the alternative description, in which it is just one central $\mathrm{CH}$ group that rotates. It was pointed out that the results directly support the calculated molecular structure at a conical intersection, which mediates the $Z-E$ isomerization of polyenes. With a more sophisticated technique, Saltiel et al. (J. Phys. Chem. Lett., 2013, 4, 716-721) confirmed this tachysterol rotamer as the main product but found two additional conformers. They believed to have seen also three previtamin D conformers, suggested to be a result of hot-ground-state reactions from the primary rotamer, and interpreted all tachysterol products to be a result of a double-bond twist (DBT), not a HT. On the basis of published circular dichroism data and consideration of other reactions, it is here shown that under these conditions hot-ground-state reactions are unimportant or even negligible and that there is practically only a single conformer of previtamin D after ring opening. All products can be easily understood on the basis of an HT-type conical intersection, which is thus further supported. Invoking a published pretwist model even rationalizes product ratios. The two twists in HT are concerted. Furthermore HT is fully consistent with the NEER principle (nonequilibration of excited rotamers) and even offers additional possibilities for conformer control.
\end{abstract}

\section{Introduction}

The $Z-E$ photoisomerization of previtamin $\mathrm{D}$ (Pre, a steroid $Z$-triene; structures are given below) to tachysterol (Tachy, the corresponding $E$-triene) was studied by our group in 1998 in Garching in a matrix (EPA: diethyl ether, isopentane, ethanol $5: 5: 1$ ) at $90 \mathrm{~K}^{1}{ }^{1}$ Because Pre was prepared in situ in the cold glass matrix by photochemical ring opening of 7-dehydrocholesterol (Dehy, a cyclohexadiene derivative; also called provitamin $\mathrm{D}_{3}$ ), it was reasonable to assume that it is a single conformer, still ring-like, with the two single bonds adjacent to the central double bond in the $c i s$ form ( $c Z c$-Pre). It was found that not only the central double bond has rotated in the product tachysterol but also an adjacent single bond (to $t E c$-Tachy). The concerted twist of such two neighboring bonds is equivalent to the rotation of a single $\mathrm{CH}$ group, which is the basis of the name Hula twist (HT). It was pointed out in ref. 1 that HT isomerization passes through an intermediate geometry (with two bonds partially twisted) that is just the calculated structure at the $S_{1} / S_{0}$ conical intersection (CI) for $Z-E$ isomerization of polyenes; ${ }^{2-7}$

Weidachst. 12, 85748 Garching, Germany.E-mail: w.fuss@mpq.mpg.de, werner@esperanto-muenchen.de; Tel: +49-89-3201807

$\dagger$ Formerly at: Max-Planck-Institut für Quantenoptik, 85741 Garching, Germany. the results are hence an experimental support of the calculated CI structure.

In 2013 the Saltiel group in Tallahassee/Florida reinvestigated this system (at $77 \mathrm{~K}$ in EPA) with a more elaborate technique and found two additional Tachy conformers: $c E c$ and $c E t$ besides the main product $t E c{ }^{8}{ }^{8}$ They believed to have seen also three Pre conformers $(c Z c, t Z c$ and $c Z t)$ as products of the Dehy ring opening. They interpreted each Tachy conformer as a result of a simple double-bond twist (DBT) of the corresponding Pre conformer; hence they concluded "DBT, not HT". ${ }^{8}$ In 2017 they investigated in the same way the photoisomerization of lumisterol (Lumi) to Pre and Tachy. ${ }^{9}$ (In Lumi and Dehy the diene chromophores form a right-handed and left-handed screw, respectively. The same difference must be assumed for the - nonplanar - triene chromophores of the two resulting $c Z c$-Pre-s.) They found from Lumi only one Pre conformer $(c Z c)$ but two (or three) Tachy conformers, whose identification seems to be less clear-cut than on starting from Dehy. They say that also in the Lumi experiment their results are more consistent with DBT than with HT.

The quantum-chemical calculations for the CI that controls $Z-E$ isomerization of polyenes ${ }^{2-7}$ are widely accepted. At this CI the molecule has twisted two neighboring CC bonds by (much) less than $180^{\circ}$; i.e., it has performed an incomplete HT, and 
branching is possible from there to complete HT, to DBT, to singlebond rotation (SBR) or internal conversion (IC) ${ }^{2-7}$ (Section 3). If it turned out that polyenes follow only DBT (and IC) but never (a concerted) HT (as claimed at the end of ref. 8), the quantum mechanical calculations should also be revised. According to Saltiel $^{8}$ the case of Pre was believed to be the most conclusive evidence for HT. It seems hence important to revisit this example.

For a critical assessment of the processes going on, let us first consider details of the previous experiments on Pre, then look once more to the predictions based on the HT-type CI (which was not mentioned in ref. 8 and 9). It will then be shown that Saltiel's results in ref. 8 are more consistent with the CI mechanism and also those in ref. 9 seem compatible with it. Also further related questions will be considered such as the NEER principle and conformer control, volume constraints and reactions in the hot ground state.

\section{Previous experiments on Pre photoisomerization}

Scheme 1 shows the structures of the compounds discussed here. The trienes differ in the orientation (s-cis or s-trans) of the single bonds in the conjugated chain. A fourth such conformer of Pre and Tachy ( $t Z t$ and $t E t$ ) is not shown; they were not detected in ref. 1, 8 and 9. The dienes and in particular the trienes are not planar. Each of the conjugated single bonds can be twisted from planarity in positive or negative helical sense; such torsions obviously influence the circular dichroism (CD).
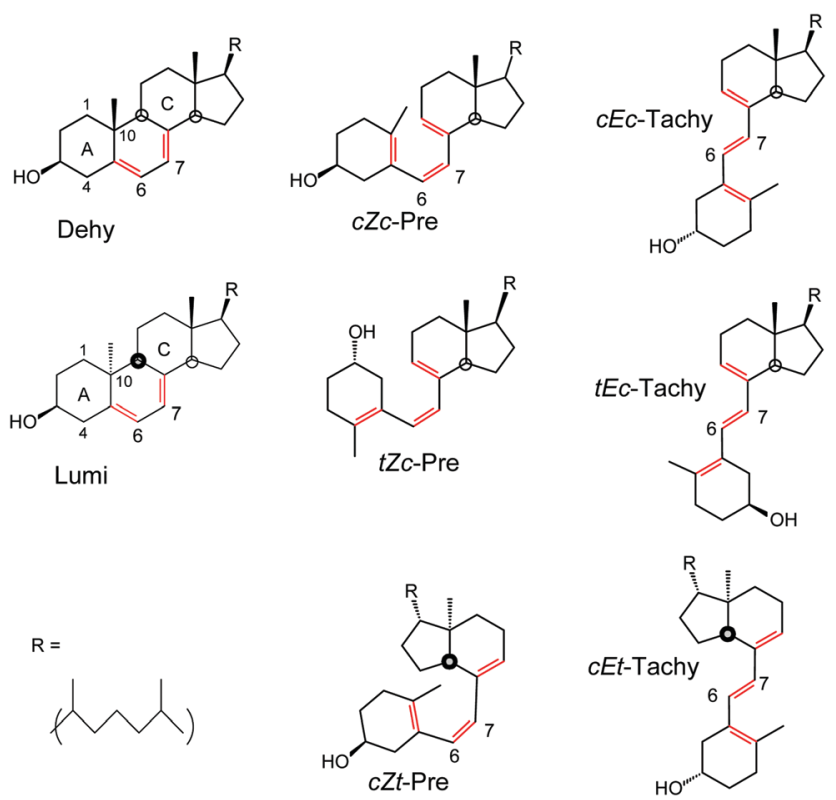

Scheme 1 Structures of 7-dehydrocholesterol (Dehy), lumisterol (Lumi), previtamin D (Pre) and tachysterol (Tachy). $c$ and $t$ denote s-cis and s-trans arrangement around the 5-6 and 7-8 single bonds. Obviously the molecules are not planar; the chromophores in Dehy and its primary ring-opening product $c Z c$-Pre form a left-handed screw $((-) c Z(-) c$-Pre), whereas in Lumi and its $c Z c$-Pre product $((+) c Z(+) c$-Pre) they correspond to a right-handed screw.
Thus one expects as primary products $(-) c Z(-) c$-Pre from Dehy and $(+) c Z(+) c$-Pre from Lumi. Furthermore, the $\mathrm{OH}$ group of the cyclohexene A ring can be equatorial or axial, which also has some influence on the torsions in the chromophores. By various quantum chemical methods Dmitrenko and coworkers ${ }^{9-12}$ calculated for these conformers of Pre and Tachy the relative energies and conformer equilibria at room temperature, structures (in particular twist angles), UV and CD spectra, mainly in the gas phase. The energy differences are small, so that solvent interactions may be more important to control which conformer dominates. On starting from a thermal Pre, there can always be uncertainties as to which conformer(s) is (are) involved. A better chance for deciding on "HT or DBT" can be expected, if one tries to prepare a single Pre conformer (and hopefully freeze it) by a ring opening reaction.

Already in the 1980s, $Z-E$ isomerization of thermal Pre was extensively investigated in a matrix of EPA and also of a hydrocarbon mixture at $92 \mathrm{~K}$ by the group of Jacobs and Havinga in Leiden, ${ }^{13-15}$ using UV, CD and NMR spectroscopy and other methods. They found the less stable $c E c$-Tachy on illuminating thermalized Pre in EPA at $92 \mathrm{~K}$; they presented arguments that under these conditions Pre was primarily in the $c Z c$ form, so that the reaction would be a DBT. ${ }^{13-15}$ After the in situ preparation of $c Z c$-Pre (according to the assumption) that photoisomerized to $t E c$-Tachy (via $\mathrm{HT}$ ), the Garching group ${ }^{1}$ reassigned thermal Pre to $t Z c$-Pre (because a different product requires a different reactant), consistent with the sign of the circular dichroism ${ }^{13-15}$ and the assignment of Dauben, ${ }^{16}$ so that the reaction was $t Z c$-Pre $\rightarrow$ $c E c$-Tachy (hence also HT) in the Leiden experiment. This assignment will be maintained here, although the Florida and Leiden groups ${ }^{8,9,13-15}$ argued in favor of "thermal Pre $=c Z c$-Pre".

It is also worth mentioning that the photoreactions of Pre and other steroid polyenes served in Leiden as important test cases for the NEER principle. This rule of nonequilibration of excited rotamers ${ }^{17-19}$ is very successful in explaining many wavelength dependences, which could thus be understood as conformer control. An example may serve for explanation (details and more examples in Section 3.2): due to the ring-like shape of its chromophore, $c Z c$-Pre can photochemically close the ring (to Dehy or Lumi), whereas the more stretched conformers cannot; all conformers can undergo $Z-E$ isomerization. Due to its spectral wing, the $c Z c$ conformer can be selectively excited at long wavelengths, so that ring closure is more important there than at shorter wavelengths. The NEER principle prevents the interconversion of different conformers during the photochemical reactions.

In the matrix experiments in Garching and Florida ${ }^{1,8,9}$ the reactant (Pre) was produced in situ by photochemical opening of the cyclohexadiene B ring of Dehy ${ }^{1,8}$ or Lumi. ${ }^{9}$ In ref. 1 it was assumed that the resulting conformer has the $c Z c$ structure, which has still a ring-like form, and $Z-E$ isomerization was found to produce the $t E c$ conformer of Tachy (see the structures in Scheme 1); from $c Z c$ to $t E c$ means rotation of two bonds, hence HT. The Florida work starting from Dehy ${ }^{8}$ confirmed $t E c$-Tachy as the main product (76\% at high conversion, using a $\mathrm{Hg}$ lamp, p. 719 left column in ref. 8) besides some $c E t$-Tachy (21\%) 
(a)

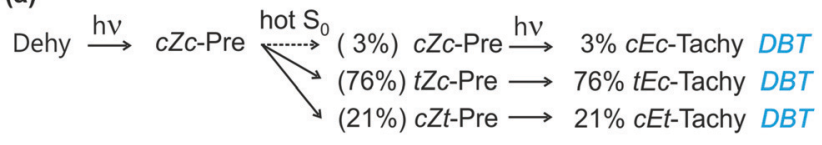
thermal Pre $=c Z c$-Pre $\stackrel{\mathrm{hv}}{\longrightarrow} c E c$-Tachy $D B T$

$$
\begin{aligned}
& \text { (b) }
\end{aligned}
$$

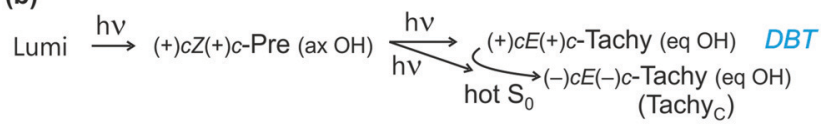

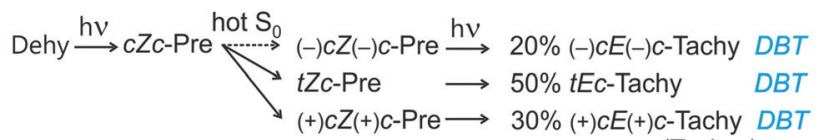

(Tachy $_{C}$ )

(c)

$$
\begin{aligned}
& \text { Dehy } \stackrel{\mathrm{h} v}{\longrightarrow} \text { cZc-Pre } \stackrel{\mathrm{h} v}{\longrightarrow} \text { cEc-Tachy DBT (attempted HT) } \\
& \text { tEc-Tachy } H T-6 \text { (main product) } \\
& \text { cEt-Tachy HT-7 } \\
& \text { thermal Pre }=t \text { Zc-Pre } \stackrel{\mathrm{h} v}{\longrightarrow} \quad c E c \text {-Tachy } \quad H T-6
\end{aligned}
$$

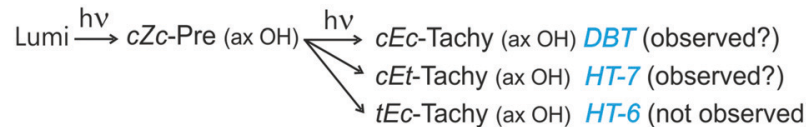

Scheme 2 The photochemical reactions of Dehy, Lumi and Pre as assigned in ref. 8 (a), ref. 9 (b) and suggested in this work (c). Where the helicity and $\mathrm{OH}$ group orientation are not given, they are assumed to be the same as in the starting material; i.e., - and eq-OH with Dehy, + and ax- $\mathrm{OH}$ with Lumi. In (a), the Tachy fractions and assignments are from p. 719 of ref. 8. The fractions were determined at high conversion with a $254 \mathrm{~nm}$ lamp and may need (small) corrections, if the fluorescence quantum yields of Tachy are taken into account. The Pre fractions are not explicitly given there. The assignment of thermal Pre is from p. 718 left column of ref. 8 . In (b), the ax and eq assignments and the helicities of the Lumi products are from p. 2339 right column in ref. 9 (also eqn (2) and Abstract). The helicity reversal via a photochemical path (not explicitly stated in ref. 9) is deduced from Fig. 7b and the hot- $S_{0}$ path is from p. 2336 bottom left. The revised percentages of the Dehy products (evaluated from absorption instead of fluorescence data) are from p. 2337 of ref. 9; the numbers $20 \%$ and $30 \%$ must possibly be interchanged. ${ }^{9}$ The final assignment of Tachy $y_{c}$ is discussed on p. 2337 top right. In (c), the first question mark after "observed" refers to the supposed ax- $\mathrm{OH}$ orientation, whereas ${ }^{9}$ prefers eq-OH. The second question mark means that "observed" is only correct with the old identification Tachy ${ }_{C}=c E t$-Tachy.

and little $c E c$-Tachy (3\%); the percentages were revised in ref. 9 but with $t E c$-Tachy still as the main product (50\%). (Also one side product, $c E t$-Tachy, was reassigned, see below.) The authors of ref. 8 also believe to have seen evidence for three conformers as products of Dehy ring opening ( $t Z c-, c Z t$ - and $c Z c$-Pre) and assign the $Z-E$ isomerization to DBT (Scheme 2a); they also assume (as in ref. 1) that $c Z c$-Pre is the primary conformer but that the other two are formed from it via hot-ground-state reactions. ${ }^{8}$ By contrast, from Lumi the authors found $c Z c$-Pre as the only ring-opening product, and subsequent $Z-E$ isomerization produced two or three tachysterol conformers, ${ }^{9}$ whose identification seems to me less clear-cut than on starting from Dehy, ${ }^{8}$ however.
The earlier in situ work ${ }^{1}$ identified the tachysterol conformers on the basis of UV spectra, which were previously assigned in Leiden $^{13-15}$ with the help of CD spectra. Saltiel and coworkers ${ }^{8,9}$ observed fluorescence in addition. This provides an additional degree of freedom, because the excitation wavelength can be varied, so that the spectra of different conformers are easier to separate. This was the basis for identifying in a thermalized mixture three conformers of tachysterol ( $t E c, c E t$ and $c E c)$, which were then also found as products of $Z-E$ isomerization in the matrix. At low temperature, tachysterol is photostable and has a good quantum yield for fluorescence. ${ }^{8}$ Unfortunately this is not the case for previtamin D. Therefore the identification of the Pre conformers is much less certain. It depends critically on the least well established fluorescence spectrum (of "Pre 290 ") and indirect evidence for its absorption (p. 719 left column in ref. 8). In the Lumi experiment ${ }^{9}$ also the Tachy conformers presented some difficulties of identification, because their fluorescence and fluorescence excitation spectra were continuous without a characteristic structure. The authors used comparison with spectra from their Dehy experiment ${ }^{8}$ and from their quantumchemical calculations. ${ }^{9}$ The Dehy-Lumi comparison caused the authors to re-assign a conformer with the preliminary name Tachy $_{\text {C }}$ (p. 2336 of ref. 9): the fluorescence spectra of the pair $(-) c E(-) c$-Tachy and Tachy ${ }_{C}$ from Dehy were similar to those of $(+) c E(+) c$-Tachy and Tachy ${ }_{C}$ from Lumi. Therefore the authors suggest that Tachy ${ }_{\mathrm{C}}$ is also a $c E c$ conformer but with opposite helicity compared with the primary conformer each time. It is not said why the original assignment ${ }^{8}$ Tachy $_{\mathrm{C}}=c E t$-Tachy seems less probable. Section 4.2 points out that the change in helicity after ring opening of Dehy is not consistent with the measurements of circular dichroism, and considerations of potentials (Section 3.1) would be better compatible with the original assignment. Therefore Schemes $2 \mathrm{a}$ and $\mathrm{b}$ present both possibilities.

The work in Garching that reported on an observation of $\mathrm{HT}^{1}$ triggered many investigations in a matrix, mainly by the Liu group in Hawaii, who found a large number of additional examples of HT isomerization, in particular in aryl substituted olefins and dienes. The results were repeatedly reviewed. ${ }^{20-27}$ The emphasis was always on the volume-saving properties of the $\mathrm{HT}$ reaction (in which only an internal $\mathrm{CH}$ group rotates out of plane). In fact, this was the original purpose of the suggestion of HT: to explain how rhodopsin can isomerize in the confines of the protein. ${ }^{28,29}$ It will here be pointed out again ${ }^{1,2,30,31}$ that an external force is not needed for HT.

Whereas Liu considers HT as a channel of higher (free) energy than DBT, it is actually a calculated minimum-energy path, ${ }^{1,2}$ consistent with its observation in cryogenic matrices. Liu initially suggested that HT is an exception to the NEER principle ${ }^{20-22}$ but modifies this statement in ref. 21 (footnote 45) and does not repeat it later. However, Saltiel seems to assume (Introduction in ref. 9) that NEER and HT are incompatible. It is here emphasized again ${ }^{2}$ that there is no conflict and that HT even provides more examples of conformer control.

The following section explains that the details of the new results of ref. 8 on Previtamin D can be understood by properties 
of an HT-type CI and that these new results support a path via such a CI instead of a DBT-type CI.

The reaction schemes suggested in ref. 8 and 9 and here are opposed in Scheme 2. The details are the subject of the following sections.

\section{Expectations from the potentials and conical intersections}

\subsection{Basics}

The conical intersection of potentials is a central concept of modern photochemistry (see, e.g. ref. 32-34). It provides a continuous and usually ultrafast path between potential surfaces. The upper cone of the $\mathrm{S}_{1} / \mathrm{S}_{0}$ CI can act as a collection funnel for the trajectories; the lower cone (together with other factors) controls the branching between recovery of the reactant (i.e., internal conversion) and formation of photoproducts. That is, the branching space (spanned by the gradient difference vector and the nonadiabatic coupling vector) are directly related to the quantum yield and product spectrum.

The longest-wavelength absorption of Pre (a substituted conjugated triene) corresponds to $\pi \pi^{*}$ excitation, populating the so-called spectroscopic or bright state $(1 \mathrm{~B}$ in the approximate symmetry $C_{2}$ of the chromophore). In the unsubstituted 1,3,5hexatriene (as in general in longer polyenes ${ }^{35,36}$ ), relaxation from there leads around a CI to the slightly lower minimum of a twoelectron excited ("dark") state $(2 \mathrm{~A})^{37,38}$ (Fig. 1). In this $\mathrm{S}_{1}$ minimum the double bonds are lengthened and the single bonds shortened (more than in the single-electron excited $1 \mathrm{~B}$ state) but the molecule is still planar (review: ref. 36). The bondorder change (usually not "reversal",8,9) hinders rotamerization in the excited state (as does also its usually short lifetime), which rationalizes the NEER principle. From the 2A minimum, the molecule leaves via a very small barrier and a $\mathrm{CI}$ to the ground state $\left(\mathrm{S}_{0}\right) .{ }^{37,38}$ According to CASSCF calculations, ${ }^{2-7}$ the molecular

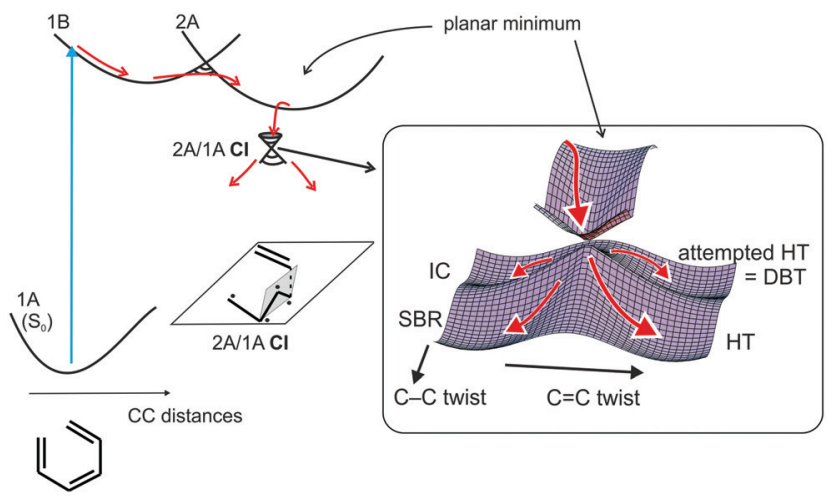

Fig. 1 Suggested potentials of 1,3,5-hexatriene. The $2 \mathrm{~A} / 1 \mathrm{~A} \mathrm{Cl}$ is displaced from the drawing plane to a direction in the branching space. The corresponding coordinates contain $\mathrm{C}=\mathrm{C}$ and $\mathrm{C}-\mathrm{C}$ twist (as indicated in the small inset), the CCC angle and a pyramidalization of the three $\mathrm{C}$ of this group. A minor barrier is indicated at the $1 \mathrm{~B} \rightarrow 2 \mathrm{~A}$ transition around the $\mathrm{Cl}$. The four exit channels lead to double-bond twist (DBT), Hula twist (HT), single-bond rotation (SBR) and internal conversion (IC). structure at this $\mathrm{S}_{1} / \mathrm{S}_{0}$ CI involves a twist of two neighboring bonds by about $60^{\circ}$ (slightly smaller in longer polyenes ${ }^{3}$ ) (Fig. 1). This structure was initially called "kink" 3 and later also "of Hulatwist type". ${ }^{2}$ The three $\mathrm{C}$ atoms in the kink are also pyramidalized (rehybridized). From the reduced CCC angle (90-100 instead of $120^{\circ}$ ), a $\mathrm{C}_{(1)}-\mathrm{C}_{(3)}$ force ("driving force") was inferred, ${ }^{2}$ which is attractive in $\mathrm{S}_{1}$ (upper cone of the $\mathrm{CI}$ ) and repulsive in $\mathrm{S}_{0}$ (lower cone). Geometrically the HT CI is (in general) less than half way to a full HT. Therefore there is no common intermediate for cis-trans and trans-cis isomerization. This explains ${ }^{2}$ why the sum of the two quantum yields is in general far below 1 in polyenes.

According to Martínez, ${ }^{39}$ this is the description of polyene cis-trans isomerization that is valid to date. It should also be noted that "the CI" actually means a minimum in the intersection space. Another minimum (of higher energy in nonpolar polyenes but lower in highly polar double-bond systems ${ }^{2}$ ) is a CI with DBT as the only twist but other coordinates involved, such as pyramidalization.

Isomerization in this simple triene begins only on departure from the (planar) $S_{1}$ minimum. To find a continuous path down to $S_{0}$, it is in general not sufficient to twist the double bond and change the bond orders again. To bring the $S_{1}$ and $S_{0}$ surfaces to intersection requires distortion along additional coordinates such as pyramidalization and single-bond twist. In the corresponding (HT-type) CI, the isomerization is less than half finished (Fig. 1). From there, on the lower cone, the wave packet can either keep its direction, supported by momentum conservation (not by the potential ${ }^{2}$ ), so that HT is completed. But it can also twist back both bonds leading back to the reactant (that is, internal conversion, IC); or turn back the single bond, resulting in DBT (called "attempted HT" in ref. 2) or the double bond, producing another conformer of the reactant (single-bond rotation, $\mathrm{SBR}^{2}$ ). Hence, one CI can lead to both HT and DBT, besides recovery of the reactant and rotamerization (Fig. 1). Due to the lack of directional force with respect to these alternatives, an external force may become decisive there, such as the inhibition of volume-demanding processes (DBT and rotamerization) by viscous media or a matrix. It should, however, be emphasized that a medium effect is not required for HT (contrary to Liu's assumption ${ }^{20-27}$ ), as shown by experiments in the gas phase and in solution (see the compilation in ref. 30 and the recent results for substituted hemithioindigos ${ }^{40}$ ) and confirmed also for Pre by calculations of dynamics in the gas phase. ${ }^{41,43}$

More recently it was pointed out that the direction of leaving from the lower cone of the CI can be predetermined: $:^{30,42}$ there can be some memory of a momentum acquired before the CI, for example via a predistortion of the molecule. In the condensed phase, this momentum conservation can last until 5-15 ps. This is the typical time of energy and momentum transfer to a solvent (Section 4.1). Examples of predistortion or momentum effects will be given in the next subsection.

Whereas most calculations have been done for unsubstituted trienes and polyenes, much less such work is available on Pre. A combination ("ONIOM") was applied to this molecule, using a high level method (CASSCF) for the chromophore and various low-level methods for the rest; ${ }^{43}$ it found that in the $\mathrm{S}_{1} / \mathrm{S}_{0}$ CI two 
neighboring bonds are twisted (by about $30^{\circ}$ ), indicating a beginning of HT. Although it was not mentioned there, the $\mathrm{S}_{1}$ in this calculation ${ }^{43}$ is probably the (dark) $2 \mathrm{~A}$ state; it is usually placed by CASSCF at lower energies than in higher-level methods. By contrast, time-dependent density functional theory (TDDFT) either tends to place this two-electron excited state at too high energies or does not find it at all. TDDFT was also applied to Pre; ${ }^{41}$ it found that $S_{1}$ is the spectroscopic (1B) state, mixed with the $2 \mathrm{~A}$ state at more distorted geometries. It also found that its $Z-E$ isomerization (in the gas phase) follows the HT pathway. ${ }^{41}$

The latter computational result was not really expected, because an HT-type CI was believed before ${ }^{2}$ to be characteristic of the intersection of the ground state with the 2A state (which valence-bond theory classifies as covalent), not with the (ionic) $1 \mathrm{~B}$ state. In the examples calculated in ref. 2 , in very polar molecules the $S_{1}$ minimum was $1 B$-like and was energetically close to $S_{0}$; a pyramidalization (which is energetically not very active in radicaloids) was sufficient to make the two states intersect. In nonpolar polyenes the $S_{1}$ minimum had $2 \mathrm{~A}$ character and was energetically far from $\mathrm{S}_{0}$, and a two-bond twist (HT) was required (in addition to pyramidalization) to reach the CI. Perhaps the criterion, when to expect a CI of either HT- or DBT-type, should be the energetic gap, not the polarity. This would also be consistent with the results for the relatively polar hemithioindigos, ${ }^{40}$ where the product spectrum seems typical of an HT CI.

For hexatriene it does not matter, whether $\mathrm{C}_{(3)}$ or $\mathrm{C}_{(4)}$ rotates out of plane in its HT-type isomerization (HT-3 or HT-4): the products would not differ, although the two paths would lead via two separate CIs. By contrast, one expects different Tachy conformers from photoisomerization of Pre, even if the latter is a single conformer (Scheme 2c): from $c Z c$-Pre, HT-6 will form $t E c$-Tachy, whereas HT-7 will lead to cEt-Tachy, besides the DBT-product $c E c$-Tachy and the recovery of the reactant from both CIs. Remarkably, no tEt-Tachy is formed by this mechanism. Its absence was interpreted by a matrix effect in ref. 8 . We can conclude that,

(1) The CI mechanism predicts just the three observed ${ }^{8}$ Tachy conformers as products from a single reactant conformer (cZc-Pre, Scheme 2c). So, it is not necessary to assume ${ }^{8}$ three separate reactant conformers.

(2) The CI mechanism does not require the assumption ${ }^{8}$ of hot-ground-state rotamerization, because all observed ${ }^{8}$ products can be formed photochemically from the primary conformer ( $c Z c$-Pre). That reactions in the hot $\mathrm{S}_{0}$ play (practically) no role is indicated by CD data (Section 4.2) and by another reaction investigated in ref. 8 (Section 4.3).

On ring opening of Lumi, the Florida group observed only one conformer of Pre, assigned (p. 2339 right in ref. 9) to $(+) c Z(+) c$-Pre with an axial OH group (Scheme 2b). (Also Lumi has a positive helicity and an axial $\mathrm{OH}$ group.) So, in contrast to Dehy as the reactant, hot-ground-state rotamerization was absent. This was tentatively attributed to a slightly smaller free space available in the EPA glass. On further irradiation they found two Tachy conformers (possibly three, if the two components in the spectrum of Tachy ${ }_{C}$ indicate two conformers, Fig. 7a and context in ref. 9). The major one was assigned (p. 2339 right column of ref. 9) to $c E c$-Tachy (equatorial $\mathrm{OH}$ ), consistent with DBT or attempted HT, except that the claimed axial to equatorial rearrangement is not predicted but conceivable on relaxation down from the CI. The minor one $\left(\right.$ Tachy $_{\mathrm{C}}$ ) was attributed in the preceding work ${ }^{8}$ to $c E t$-Tachy, which would indicate HT-7, starting from Dehy or from Lumi. The authors of ref. 9 did not give any reason why they abandoned this assignment; they now prefer $(-) c E(-) c$-Tachy with equatorial $\mathrm{OH}$ for Tachy $\mathrm{C}_{\mathrm{C}}$, an assignment consistent with their spectra, in particular when compared $^{9}$ with those from the Dehy experiment.

According to Fig. 7b of ref. 9, also Tachy ${ }_{C}$ can form directly from $c Z c$-Pre (generated from Lumi). If it is $c E t$-Tachy, this corresponds to HT-7, which involves rotation of the $\mathrm{C}=\mathrm{C}$ bond by about $180^{\circ}$ and of an adjacent $\mathrm{C}-\mathrm{C}$ bond by about $150^{\circ}$. In the case of helicity inversion (i.e., if Tachy ${ }_{C}=(-) c E(-) c$-Tachy), a twist by $90-130^{\circ}$ of two single bonds is necessary besides the $\mathrm{C}=\mathrm{C}$ bond rotation (dihedral angles from Fig. $\mathrm{S} 7$ with Tables in the ESI of ref. 9). This seems like a close relative of HT. However, quantum chemistry should check whether such a CI exists and is accessible. (A two-step process, DBT followed by ground-state rotamerization, is not likely: why should a statistical process twist just two $\mathrm{C}-\mathrm{C}$ bonds?) On further irradiation the authors also observed a slow conversion, $(+) c E(+) c$-Tachy $\rightarrow$ Tachy $_{\mathrm{C}}$ (Fig. 8a and context in ref. 9). If Tachy ${ }_{C}=c E t$-Tachy, this would be a single-bond rotation, allowed by an HT-type CI (Fig. 1). The authors instead assumed a hot-ground-state rearrangement (helicity reversal) (Scheme 2b). It is not clear, however, why such a reaction should not produce other conformers as well, besides the one with opposite helicity. It is shown in Section 4 that reactions in the hot $S_{0}$ are generally minor or even negligible in Pre.

To summarize, I would interpret the isomerization of $c Z c$-Pre generated from Lumi as follows: (1) the main product, $(+) c E(+) c-$ Tachy, forms by DBT, which can be understood as an attempted HT. However, its large yield is surprising, although still consistent with an HT CI. (2) If the minor product is $c E t$-Tachy, it is a direct support for an HT CI. (3) If the minor product is $(-) c E(-) c$-Tachy, produced directly from the ring-opening product $(+) c Z(+) c$-Pre, the involved correlated twists of a double bond and two single bonds require a new type of CI. Such a CI would need quantum chemical confirmation, although it seems closely related to an HT CI. (4) cEtTachy (old assignment of Tachy $_{\mathrm{C}}$ ) can form from the main product $c E c$-Tachy by SBR via an HT CI, and $(-) c E(-) c$-Tachy by a twofold $\mathrm{C}-\mathrm{C}$ rotation possibly via the postulated CI. A selective twofold bond rotation via a hot-ground-state reaction seems not likely. (5) A pure DBT mechanism (via a DBT-type CI) would give rise to only one Tachy conformer.

\subsection{Barriers, pretwist, conformer control and the NEER principle}

By considering the pretwist in the reactants, even the product ratios found in ref. 8 can be made qualitatively plausible: it was observed that in substituted diphenyl-butadienes photochemical $Z-E$ isomerization by HT-1 was by far preferred over HT-2. ${ }^{44,45}$ This was explained in ref. 30 by a predistortion effect and extended to many more examples in ref. 42 . If in competing ultrafast reactions 
such as HT- 1 and HT-2 the molecule is predistorted in $\mathrm{S}_{0}$ in a direction containing one of the photochemically active coordinates (twist of one of the single-bond neighbors of the double bond), vertical excitation puts the wave packet to a steep slope declining in just this direction; the coordinate should at the same time be Franck-Condon active. ${ }^{42}$ The slope accelerates the molecule towards the pretwist. When the wave packet arrives in a branching region, this momentum helps in selecting the right CI (HT-1 over HT-2) or the right exit direction in a given CI (Fig. 1). A similar acceleration is observed, if the pretwist eliminates a small barrier that would exist in a planar unhindered molecule and that would slow down the reaction. ${ }^{30,42}$

In planar trienes and longer polyenes twist coordinates are not Franck-Condon active: as said above, excitation changes mainly the bond orders so that differences of $\mathrm{C}-\mathrm{C}$ and $\mathrm{C}=\mathrm{C}$ bond lengths are reduced. But the molecule remains planar in the $\pi \pi^{*}$ and $\pi^{*^{2}}$ (1B and 2A) states. However, upon excitation of the nonplanar trienes Pre or Tachy, the bond-order increase of the $\mathrm{C}-\mathrm{C}$ bonds tries to reduce their twist. That is, the coordinate becomes Franck-Condon active. Upon excitation, the wave packet is first accelerated towards planarity; but already after half a vibrational period its direction is reversed.

According to force field and other calculations, ${ }^{9,12}$ in $(-) c Z(-) c$-Pre (equatorial $\mathrm{OH}$ ) the $5-6 \mathrm{C}-\mathrm{C}$ bond is much more twisted than the 7-8 bond, so that one expects HT-6 to dominate over HT-7. This fits neatly to the ratio of $t E c$ - and cEt-Tachy products (76 versus $21 \%^{8}$ or 50 versus $30 \%{ }^{9}$ ), when the in situ Pre (assumed here and in ref. 8 to be the primary product conformer, $(-) c Z(-) c$-Pre) was illuminated by a mercury lamp. ${ }^{8}$ The low yield $\left(3 \%{ }^{8}\right.$ or $\left.20 \%{ }^{9}\right)$ of the DBT product $c E c$-Tachy conforms with the general experience (see, e.g. ref. 30) that HT is preferred in nonpolar polyenes, whenever it is not hindered. It may also be caused by hindering DBT (also on an attempted HT path) by the constrained volume.

On starting from Lumi, one expects $(+) c Z(+) c$-Pre with axial $\mathrm{OH}$. According to the calculations, ${ }^{9}$ the dihedral angles of the two single bonds are still comparable in magnitude, although the twist of the 7-8 bond is considerably increased and that of the 5-6 bond decreased compared to $(-) c Z(-) c$-Pre from Dehy. The pretwist model would then predict a higher ratio of $c E t$-Tachy over $t E c$-Tachy. In fact, the observed ratio (if Tachy ${ }_{\mathrm{C}}=$ $c E t$-Tachy) is larger, but much larger than expected: the $t E c$ conformer was probably not observed at all. So, either the pretwist model is not sufficient in this case, or the distortions in the EPA glass are much larger than those calculated for the gas phase. A difficulty in checking the pretwist model with the Lumi data is that the identification of the conformers seems to me less clear-cut than in the Dehy case.

This discussion shows that the idea of conformer control (made possible by the NEER principle) is not only fully compatible with the HT-CI mechanism (Saltiel seems to assume inconsistency ${ }^{9}$ ) but even offers more possibilities, because the $\mathrm{C}-\mathrm{C}$ bond twist also gives some control. It seems therefore interesting to consider more examples.

(1) The Saltiel group found an example of conformer control, which is of practical interest for the synthesis of (pre)vitamin D and hydroxyl derivatives ${ }^{46}$ for which the predistortion principle provides a suggestive explanation: ${ }^{42}$ Whereas the main Tachy conformer $(t E c)$ has an $E-Z$ isomerization quantum yield of only $\varphi_{E Z}=0.12$, irradiation in a long-wavelength shoulder (probably belonging to $c E c$-Tachy) achieves $\varphi_{E Z}=0.42 .{ }^{46}$ Indeed $c E c$-Tachy is more pretwisted than $t E c$-Tachy. ${ }^{9}$

(2) The conformer ratio of the Tachy product was found in ref. 8 to depend slightly on the progress of conversion. This was suggested to indicate a matrix site effect, without detailed explanation. In fact, the pretwist of $c Z c$-Pre - and thus the probability ratio of HT-6 over HT-7 - may slightly depend on the environment.

(3) In the same way one can consider the $t Z c$-Pre conformers, which according to density functional theory and molecular dynamics ${ }^{9,11,41}$ in the gas phase dominate in a thermal distribution at room temperature and certainly even more in the cold matrix. (Whereas the equilibrium might be shifted by the solvent, Section 4.3 presents experimental evidence that $t Z c$-Pre is the lowest-energy conformer in the EPA matrix.) According to the calculations ${ }^{9}$ the difference between the twist angles of the 5-6 and 7-8 bonds is greater in these conformers than for the $c Z c$ conformers, so that one can also expect a higher preference for the HT-6 product $c E c$-Tachy. The Florida group even observed $c E c$-Tachy as the only product of thermalized Pre; ${ }^{8}$ that is, of $t Z c$-Pre, as just stated.

(4) Conformer control has often been invoked to explain wavelength dependences. A famous case is that the quantum yield of the Pre $\rightarrow$ Tachy isomerization (in solution near room temperature) exhibits a sudden change within a small wavelength range: whereas in the long-wavelength wing there was only ring closure to Dehy and Lumi, ascribed to the $c Z c$-Pre conformer, $Z-E$ isomerization is observed in addition only above a certain photon energy ${ }^{47}$ (for differences with small model compounds, ${ }^{48}$ see below). The original work ${ }^{47}$ supposed a difference of photochemistry from the lower-lying dark (2A) state and the spectroscopic (1B) state. The problem with this interpretation is that from the higher potential the molecule must in any case pass the $S_{1}$ potential to reach $S_{0}$.

According to an alternative model (see e.g. ref. 49 and 50 and literature quoted there) the ratio of quantum yields for ring closure and $Z-E$ isomerization simply reflects the ratio of absorptions of $c Z c$-Pre and $t Z c$-Pre. The simplest such conformer-control models assumed that $c Z c$-Pre closes the ring as the only reaction. However, as again found in ref. 1, 8 and 9, also $c Z c$-Pre isomerizes its central double bond with good yield. It is entirely conceivable that the quantum yield of this reaction is similar to that of the other conformers. In this case there would be no wavelength dependence of Tachy formation (if there is no reaction-specific barrier, as assumed in these models); examples are the small model molecules, ${ }^{48}$ see the end of this section. Indeed, when a single Pre conformer $(c Z c)$ was prepared in situ in the cold EPA matrix, the quantum yield for Tachy $(t E c)$ decreased by a factor of 1.6 from 293 to $295 \mathrm{~nm}$ (Table 5.1 in ref. 51, mentioned already in ref. 52, where also the different wavelength thresholds are discussed). Hence the quantum yield jump must (also) have an internal cause, present in a single conformer. 
In ref. 52 the sudden change was ascribed to a barrier for $Z-E$ isomerization, whereas the ring closure is (nearly) barrierless; only the smooth part of the wavelength dependence was attributed to conformer control. These ideas are supported by calculation of the conformer spectra, ${ }^{9,11,41,53}$ which showed no sudden change of absorption cross sections. The group in Leiden pointed out already in 1981 that also the wavelength dependence of vitamin D $Z-E$ isomerization (where the conformer distribution is much simpler than in Pre) cannot be understood by conformer control alone. ${ }^{54}$ They also invoked a barrier in the excited state, which is overcome by a higher photon energy.

It seems appropriate to have a closer look at the barriers, also in view of the control by the pretwist.

A barrier seems to be located at the exit from the (spectroscopic) $1 \mathrm{~B}$ state to the $2 \mathrm{~A}$ state around the $1 \mathrm{~B} / 2 \mathrm{~A}$ CI (Fig. 1): leaving $1 \mathrm{~B}$ requires a minor activation energy in the steroid dienes and some trienes. This can be concluded from the fluorescence, which (1) originates from the spectroscopic state, as indicated by the emission spectra, which show no gap between absorption and emission for Dehy (see ref. 55, 56 and quoted literature) and for some trienes (ref. 8 and 9 and quoted literature), and which (2) has a much improved quantum yield at low temperature (see ref. 8, 55 and 56 and quoted literature). The barrier seems higher in the $E$ isomer (Tachy), which is photochemically stable at low temperature, ${ }^{8,9}$ in contrast to the $Z$ isomer (Pre), in which $Z-E$ isomerization still takes place. Whereas ring opening of unsubstituted cyclohexadiene is barrierless all along the path, the $1 \mathrm{~B} \rightarrow 2 \mathrm{~A}$ transition in Dehy requires activation $\left(4-10 \mathrm{~kJ} \mathrm{~mol}^{-157}\right)$. This is expected, because the shift towards Franck-Condon active coordinates (that include $\mathrm{C}=\mathrm{C}$ torsion) of the $\pi^{*^{2}}(2 \mathrm{~A})$ potential is larger than that of $\pi \pi^{*}(1 \mathrm{~B})$. As the $\mathrm{C}=\mathrm{C}$ twist is hindered by ring fusion, the $1 \mathrm{~B}-2 \mathrm{~A}$ intersection is moved up in Dehy. Similarly in the trienes with their bondorder change in the excited states, the pretwist lifts the $2 \mathrm{~A}$ state more than the $1 \mathrm{~B}$ state, thus generating (or raising) the barrier, if plotted versus bond lengths as in Fig. 1 (Fig. 2 left). However, if plotted versus torsion as in Fig. 2, right-hand side, the increased pretwist lifts $2 \mathrm{~A}$ versus $1 \mathrm{~B}$, so that the intersection is lowered. A calculation would be desirable to decide which effect is greater. Above, momentum effects (not activation energies) were invoked for the interpretation that processes that go to the direction of the pretwist are preferred.

After localization of the barrier at the $1 \mathrm{~B} / 2 \mathrm{~A} \mathrm{CI}$, one can now figure out (better than in the barrier model ${ }^{52}$ ) the origin of the

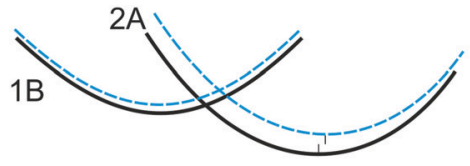

CC distances

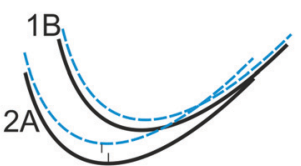

torsion angles
Fig. 2 Expected effect of the $\mathrm{C}-\mathrm{C}$ twist: due to increased bond order, torsion destabilizes the doubly excited (2A) state more than the $\pi \pi^{\star}(1 \mathrm{~B})$ state. In a cross section along CC distances, the surface crossing and $1 B / 2 A$ barrier are raised thereby, but lowered along a torsion coordinate. wavelength dependence of the ( $c Z c$-) Pre photochemistry: in the wavelength region near the jump of quantum yields, excitation leads directly into the branching region at the 1B/2A CI (Fig. 3). The longest wavelengths $\left(\lambda_{3}\right)$ still reach a barrierless path, which leads to a "pericyclic" $2 \mathrm{~A}$ minimum and CI towards ring closure. Larger photon energies $\left(\lambda_{1}\right)$ can in addition activate also $Z-E$ isomerization. With an intermediate wavelength $\left(\lambda_{2}\right)$ the isomerization barrier can be overcome only with additional thermal activation (dotted arrow). This description is close to the barrier model, ${ }^{52}$ where the $2 \mathrm{~A}$ state was not explicitly used and where overcoming of the barrier was by the excess energy, as in a thermal process, instead of a ballistic process as suggested here. It is also close to the original interpretation, ${ }^{47}$ where direct excitation of $1 \mathrm{~B}$ and $2 \mathrm{~A}$ states was assumed (but also that they differ in the products). In fact, near the $1 \mathrm{~B} / 2 \mathrm{~A} \mathrm{CI}$ with its strong vibronic interaction, the $2 \mathrm{~A}$ "dark" state borrows intensity from $1 \mathrm{~B}$, so that the absorption cross sections to the two states are comparable in this region.

The quantitative evaluation of branching ratios in ref. 52 can be taken to the present model. In ref. 47 the Pre fluorescence was assigned to emission from the $2 \mathrm{~A}$ state. Because its measured lifetime ( $2 \mathrm{~ns}$ ) is much longer than the $c Z c$-Pre lifetime in the $2 \mathrm{~A}$ minimum ( $\leq$ ring opening time of Dehy $\approx 1.8 \mathrm{ps}^{56}$ or at low temperature not longer than $5 \mathrm{ps} ;{ }^{71} c Z c$-Pre has this "pericyclic" minimum in common with Dehy), this fluorescence must be

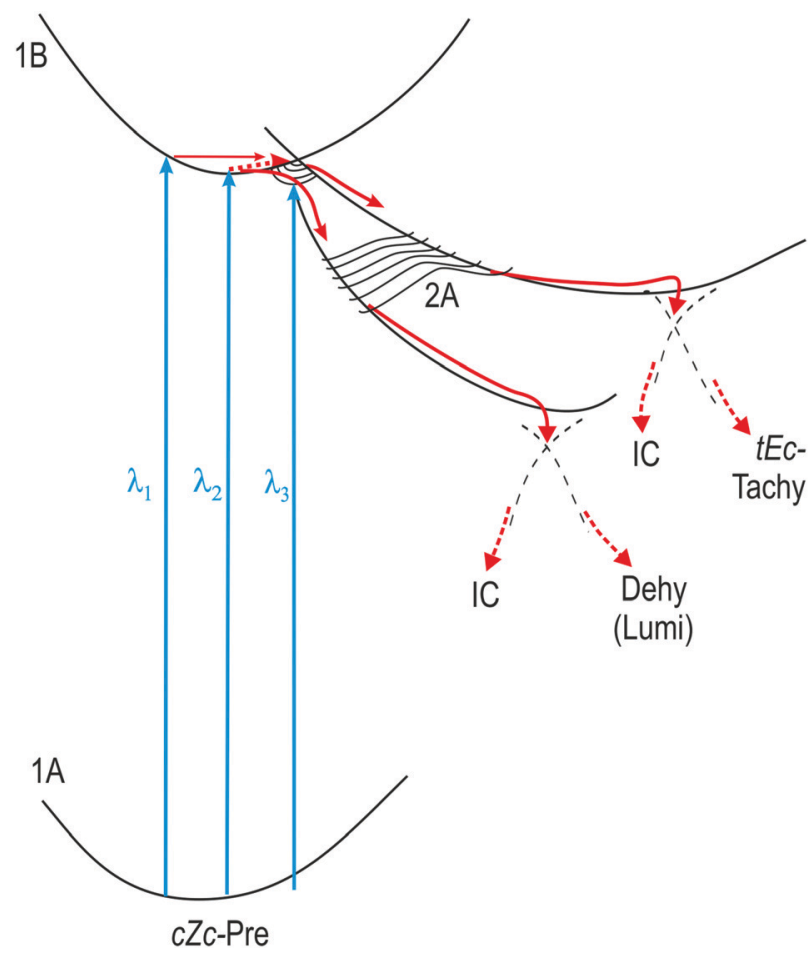

Fig. 3 Suggested interpretation of a wavelength dependence in a single (cZc-) Pre conformer: two valleys on the $2 \mathrm{~A}$ surface lead to ring closure (Dehy or Lumi, depending on the Pre helicity) or Z-E isomerization (tEc-Tachy), respectively. In the branching region $(1 \mathrm{~B} / 2 \mathrm{~A} \mathrm{Cl})$ one path is barrierless, the other (behind the drawing plane) climbs over a small barrier. Only the former is reached by long wavelengths $\left(\lambda_{3}\right)$. Dotted arrow: thermal activation, IC: internal conversion. 
assigned to another conformer, $t Z c$-Pre. Upon photochemical ring opening of Dehy, the molecule reaches via a 1B/2A CI (from the right-hand side of the $2 \mathrm{~A}$ curve of Fig. 3 , not shown) the same pericyclic minimum that is shown in Fig. 3 (in contrast to the suggestion in ref. 47). Nevertheless there is no wavelength effect in Dehy, ${ }^{47}$ because from its $1 \mathrm{~B} / 2 \mathrm{~A}$ CI the $Z-E$ valley is not accessible.

The model attributes the barrier to the $Z-E$ isomerization, consistent with its yield that strongly drops towards longer wavelengths. At the same time, the ring closure yield increases, with the sum of yields found to be wavelength independent, ${ }^{47}$ consistent with the competitive nature of the two reactions. By contrast, while investigating two smaller trienes instead of Pre, Dauben's group found a wavelength independent $Z-E$ isomerization. ${ }^{48}$ Obviously these model compounds meet no barrier in this reaction. (According to Fig. 2 the barrier height can depend on small geometric details.) By contrast, ring closure was found in these compounds to increase strongly towards longer wavelengths; ${ }^{48}$ this dependence seems consistent with a pure conformer control, where the $c Z c$ absorption dominates in the red wing of the spectrum. (The authors assume an electronic reason. ${ }^{48}$ ) It is also worth noting that in this example all conformers, including $c Z c$, have the same $Z-E$ yields, contrary to some assumptions mentioned above.

It can be assumed that (as usual ${ }^{36}$ ) the branching space of the $1 \mathrm{~B} / 2 \mathrm{~A} \mathrm{CI}$ is spanned by similar coordinates to the last $\mathrm{CI}$. That is, the $\mathrm{C}=\mathrm{C}$ and $\mathrm{C}-\mathrm{C}$ torsions dominate in it. Interestingly, in this case the two paths differ in the relative sign of the two torsions: the barrierless one (ring closure) follows a conrotatory motion, as expected for a Woodward-Hoffmann allowed ring closure, whereas the activated path uses disrotatory (mirrorsymmetric) motion of the two bonds, as expected for Hula twist.

\section{Practically no hot-ground-state reactions: evidence from circular dichroism and various reactions}

4.1. Reactions in the hot ground sate: expectations and some observations. Hula twist is concerted

Reactions in the hot $\mathrm{S}_{0}$ are a frequent phenomenon in gas-phase photochemistry. After the return to the ground state of the reactant or product, the photon energy is fully released (reactant) or nearly so (product) as thermal (vibrational) excess energy. The correspondingly high temperatures lead to thermal reactions in the electronic ground state. They are expected to approach a (high-temperature) thermal equilibrium but do not go beyond (as also confirmed by calculation for Dehy ring opening in the gas phase ${ }^{41}$ ). Such reactions are easy to distinguish from true photochemical reactions (i.e. those following the slopes on the potential from $S_{1}$ to $S_{0}$ ), see e.g. the discussion in ref. 58: in contrast to the latter, a buffer gas suppresses them partially, and in the condensed phase they are typically only detectable in traces; only with small molecules such as ethylene they still contribute substantially to the products $\left(\mathrm{H}\right.$ and $\mathrm{H}_{2}$ elimination in this example $\left.{ }^{58}\right)$. The crucial parameters are the initial temperature and the rate of cooling compared to the reaction rate. In a nonpolar condensed phase, cooling (reduction of the temperature difference by a factor of e) takes 5-15 ps; it can be faster, if there are polar interactions or hydrogen bridges (see ref. 59-61 and literature quoted there). If there is any hot- $\mathrm{S}_{0}$ reaction in the condensed phase, it must take place before. This can happen, if the initial temperature is high enough, for example in small molecules, where the excess energy is distributed over only few degrees of freedom; or if the activation energy for the reaction is small enough, for example, in rotamerization.

The photochemical ring opening of 1,3-cyclohexadiene (CHD) to $Z$-hexatriene may serve as an example. After excitation at $267 \mathrm{~nm}(4.6 \mathrm{eV})$ and internal conversion, CHD has a calculated vibrational temperature of $2400 \mathrm{~K} .{ }^{62}$ In the gas phase (i.e. without cooling), ring opening goes to completion. In solution, the ring opens with $42 \%$ quantum yield and, as concluded from substituted derivatives, on a purely photochemical (conrotatory) path. Obviously the increase of the yield in the gas phase is due to thermal (disrotatory) ring opening. (This was not recognized in the reviews. ${ }^{56,63}$ ) That is, hot-ground-state ring opening is completely suppressed in solution in this example. The product $c Z c$-hexatriene has a calculated initial temperature of $2050 \mathrm{~K} ;^{62}$ it was observed to rotamerize in solution, although not to completion (summary in ref. 55-57). Probably the much smaller activation energy helps that this hot- $\mathrm{S}_{0}$ reaction is much less suppressed.

Previtamin D has a much larger number of vibrational degrees of freedom; if most of them absorb the excess energy after ring opening of Dehy, it is expected to have a much smaller initial temperature than hexatriene. If intramolecular vibrational energy redistribution is incomplete at early times, the temperature of the chromophore may still be high enough to allow rotamerization of the ring-opening product Pre. Such a possibility was considered in ref. 55-57 and in ref. 8 and 9. A nonadiabatic molecular dynamics calculation in the gas phase (i.e., without cooling) found $t Z c$-Pre after Dehy ring opening already near 0.7 ps and a conformer equilibrium around 4 ps. ${ }^{41}$ The following two sections present experimental evidence that in the condensed phase rotamerization is by far less extensive than claimed in ref. 8 and 9 or may be nearly negligible. If so, it is suppressed by the combined intraand intermolecular energy redistribution (cooling), which seems efficient in the large molecules.

One may also ask why the authors of ref. 8 believe to have seen extensive rotamerization in the hot $S_{0}$ of Pre produced from Dehy but not if produced from Lumi ${ }^{9}$ (although the authors point to a different free space in EPA in this case ${ }^{9}$ ) or in the Tachy products upon irradiation of Pre; ${ }^{8,9}$ they have the same molecular size and excess energies. One such example is considered in Section 4.3.

A characteristic difference between photochemical and thermal reactions is the following:

Whereas two photochemical reactions

$$
\begin{aligned}
& \text { conformer A } \rightarrow \text { conformer B } \\
& \text { conformer B } \rightarrow \text { conformer A }
\end{aligned}
$$


are easily conceivable (e.g. SBR via an HT CI) and do not contradict each other, thermally one would obtain (at high temperature) one and the same conformer mixture from both reactants. For illustration: if the conformers were isoenergetic, a hot- $\mathrm{S}_{0}$ reaction would produce $\mathrm{A} / \mathrm{B}$ ratios approaching the equilibrium value of $1: 1$ with both reactants, whereas the photochemical process can produce B from A and A from B. A clear-cut example of the photochemical type is (conformercontrolled) rotamerization of butadiene in a cryogenic matrix: ${ }^{64}$ Irradiation at $214 \mathrm{~nm}$ (s-trans) produced s-cis, whereas excitation near $230 \mathrm{~nm}$ (s-cis) resulted in s-trans; by contrast, the product composition should be very similar (i.e. near equilibrium) with the two wavelengths, if it were a matter of a ground-state reaction (as supposed by Saltiel (p. 2336 of ref. 9)). (Liu interprets this and other examples as an HT process. ${ }^{20-27}$ ) More recent examples of the photochemical type are the substituted hemithioindigos of ref. 40, where the conformer pairs $\mathrm{A} / \mathrm{B}$ and $\mathrm{C} / \mathrm{D}$ could individually be rotamerized as in eqn (1) and (2).

This criterion can be used to answer whether Hula twist is concerted: Saltiel repeatedly ${ }^{8}$ suggested that observed cases of HT are actually photochemical DBT followed by rotamerization in the hot ground state. If this were right, one would observe (nearly) the same (equilibrium) conformer mixture of Tachy, starting from different Pre conformers. Actually one observes ${ }^{1,8}$ in the cold EPA

$$
\begin{aligned}
& \text { in situ Pre } \rightarrow t E c \text {-Tachy (predominantly) } \\
& \text { thermal Pre } \rightarrow c E c \text {-Tachy (exclusively). }
\end{aligned}
$$

The latter is a less stable conformer of Tachy. It should not dominate (or even be the exclusive product) in a thermal reaction. (Many analogous cases have been reported by Liu. ${ }^{20-27}$ ) Hence HT is concerted, in agreement also with the HT-CI mechanism. An asynchronous (but concerted) case was reported for stilbene. ${ }^{31}$

To state it in other words: the pair of eqn (3) and (4) implies both: that there is no equilibration of rotamers in excited Pre (i.e., HT obeys NEER) and that there is no equilibration of conformers in ground-state Tachy (i.e., HT is not a two-step process but is concerted).

According to the next section, measurements of the circular dichroism in ring opening of Dehy indicate that reactions in the hot ground state of Pre are by far less important than claimed in ref. 8 and 9. Section 4.3 suggests they are even practically negligible, if one can conclude by analogy with thermalized Pre.

\subsection{Circular dichroism}

7-Dehydrocholesterol (Dehy) has a negative circular dichroism (CD) (see, e.g. ref. 65); that is, the absorption in its first UV band is larger, if incident circularly polarized light is right-handed than if it is left-handed. This is due to the left-handed helix, formed by its diene chromophore, in agreement with the general rule for nonplanar dienes (see, e.g., chapter 11 of ref. 65). This rule can be extended to trienes (theoretical support in ref. 66 and 67, measured in cyclic trienes in ref. 68). For Pre and Tachy conformers the CD spectra were calculated by Dmitrenko et al. ${ }^{9}$ In the region of their $\mathrm{UV}$ bands, the $\mathrm{CD}$ of $(-) c Z(-) c$-Pre is negative (probably with larger magnitude than in Dehy, because of the larger twist) and positive for the $(+) t Z(-) c$ and $(-) c Z(+) t$ conformers, which result from the former by rotamerization. Of course, it is also positive for $(+) c Z(+) c$-Pre, the product of Lumi ring opening. Obviously the $\mathrm{CD}$ can help decide which conformer is produced upon ring opening and whether there is any hotground-state reaction thereafter.

A first hint is given by preliminary experiments in Leiden (end of chapter 6 in ref. 15), where upon ring opening of Dehy (Lumi) in a cold EPA matrix the CD stayed negative (positive). It would be desirable to repeat their experiments and isolate the absorption and CD spectra of the $c Z c$-Pre conformers and determine their yields. But valuable evidence is already provided by a room-temperature measurement of the CD change on Dehy ring opening. ${ }^{69}$ As it was time resolved, the effect of the primary products could be isolated, undisturbed by later reactions. The resulting $\mathrm{CD}$ changes are shown in Fig. 4. The photolysis (pump) pulse immediately makes the CD signal more negative, probably due to an excited-state contribution; ${ }^{69}$ the peak then decays with a time constant of 1.1 to 1.9 ps to a constant negative plateau, which lasts at least $18 \mathrm{ps}$. The time constant agrees well with that for ring opening found by transient absorption. ${ }^{55,57}$ The pedestal represents the contribution of the product to the CD. This contribution is obviously negative, as expected for the primary conformer $(-) c Z(-) c$-Pre with its increased negative $\mathrm{CD}$, whereas the $t Z c$ and $c Z t$ conformers ${ }^{8,9}$ or the $c Z c$ form with opposite helicity ${ }^{9}$ with their positive $\mathrm{CD}^{9}$ would give rise to a positive pedestal (= difference of CD after and before irradiation), if present with substantial quantities; also the depletion of Dehy (with has a negative $\mathrm{CD}$ ) gives a positive contribution. Obviously there is no substantial rotamerization during this time at room temperature in solution. Even with the limited signal-to-noise ratio, one can rule out that $97 \%$ of

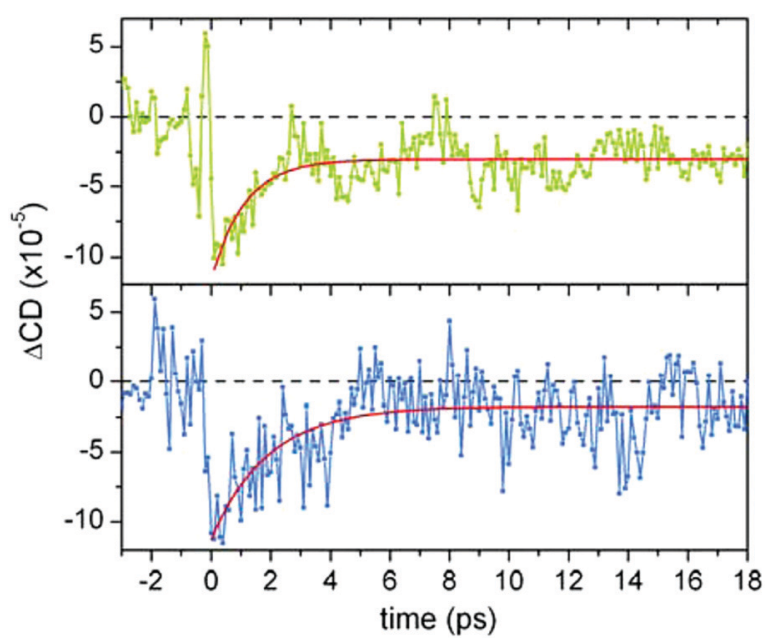

Fig. 4 Time-resolved circular dichroism (CD) upon photochemical ring opening of Dehy, measured in ref. 69 with $300 \mathrm{~nm}$ (upper panel) and $285 \mathrm{~nm}$ for photolysis and probing. The shown $\triangle C D$ is the measured CD minus that before irradiation, so that a negative value indicates that the $C D$ has become more negative than that of Dehy. Reproduced with permission of ACS. 
the primary conformer rearrange to either $(+) t Z(-) c$-Pre or $(-) c Z(+) t-$ Pre $^{8}$ or $80 \%$ to either $(+) t Z(-) c$-Pre or $(+) c Z(+) c$-Pre. ${ }^{9}$

It may be argued that the CD measurements are consistent with rotamerization of a small fraction of the claimed high percentages; i.e., that hot-ground-state isomerization after ring opening of Dehy is not zero, although much less important than suggested in ref. 8 and 9. If, for example, only a fifth of the $80 \%$ (claimed in ref. 9) does rotamerize, $16 \%$ would be rearranged and $84 \%$ of the primary conformer would remain in the $c Z c$ form. Even in this case most of the $t E c$-Tachy $\left(50 \%{ }^{9}\right)$ must have been produced directly from $c Z c$-Pre, not via a two-step process (hot-ground-state reaction + DBT). Hence one must also drop the idea $^{8,9}$ that each detected Tachy conformer has a precursor (Pre conformer) of its own with equal abundance.

The CD results also limit the yield of $(+) c Z(+) c$-Pre (with inverse helicity of the primary conformer) to much less than $30 \%$ (to perhaps a fifth of it, as suggested above). This number was implied by the revised identification of Tachy $_{C}$ with $(+) c E(+) c$-Tachy, the measured yield of Tachy ${ }_{C}$ and the assumption of DBT isomerization from Pre to Tachy. ${ }^{9}$ Either one should change the assignment of Tachy ${ }_{\mathrm{C}}$ again (perhaps back to $c E t$ Tachy, which can be formed from the primary $c Z c$-Pre) or one must find another precursor of $(+) c E(+) c$-Tachy (perhaps the primary $c Z c$-Pre and a new type of CI, see Section 3.1).

In the conical intersection mechanism all Tachy conformers observed from Dehy in ref. 8 can result from $c Z c$-Pre alone. If Tachy $_{\mathrm{C}}=c E t$-Tachy (as in ref. 8 ), also the products of $\mathrm{Lumi}^{9}$ are compatible with this mechanism with a single precursor (Section 3, Scheme 2c).

If there are any hot-ground-state reactions in the Dehy/Pre system, they must take place in a time clearly shorter than 5 ps: this was the cooling time measured also in this system by transient absorption (see, e.g. ref. 70).

At much longer times (after cooling) a thermally activated rotamerization was found, however: ${ }^{70,71}$ It has a time constant of $125 \mathrm{ps}$ in ethanol at ambient temperature. The measured activation energy $\left(16 \mathrm{~kJ} \mathrm{~mol}^{-171}\right)$ infers that it is frozen in the cold matrix. The time dependent absorption increased substantially during this relaxation over several hundred picoseconds, ${ }^{71}$ as expected for a (predominant) $c Z c$-Pre $\rightarrow t Z c$-Pre rotamerization, because the latter conformer has a twice larger absorption cross-section than the former. ${ }^{9}$

\subsection{Cold thermalized Pre is $t Z c$-Pre, not $c Z c$-Pre}

If Pre prepared in situ photoisomerizes to (mainly) $t E c$-Tachy ${ }^{1,8}$ but thermal Pre (thermalized at low temperature) isomerizes to a different product ( $c E c$-Tachy), ${ }^{8,13-15}$ the reactants must also be different, as already argued in ref. 1 . That is, if the in situ product is $c Z c$-Pre (Section 4.2), thermalized Pre cannot be the $c Z c$ conformer. (See Scheme 2 for the assignments of this work and of the Florida group.) Unnoticed by the Florida authors, just their work ${ }^{8}$ provides strong evidence that it is in fact not $c Z c$-Pre: by the fluorescence detection they found that $c E c$-Tachy is the only photoisomerization product formed from thermalized Pre, the irradiation mixture forming a strict two-component system. ${ }^{8}$ But from $c Z c$-Pre one would also expect the ring-closure products Dehy and Lumi (from $(-) c Z(-) c$ and $(+) c Z(+) c$, respectively), which both fluoresce efficiently at the low temperature (see ref. 8, 9, 55 and 56 and literature quoted there) and would be easily detected, also because they show a characteristic vibrational structure. (The quantum yields for ring closure were reported to be 1.5 to $3 \%$ for the room-temperature conformer mixture. ${ }^{17}$ They would be by a factor larger, if referred to pure $c Z c$-Pre.) The fact that they were not found implies that the thermalized Pre is not $c Z c$ but must be $t Z c$. The alternatives $c Z t$-Pre and $t Z t$-Pre have too high energies. ${ }^{9,10}$

A similar but independent piece of evidence that the thermalized Pre is not $c Z c$ can be taken from the UV and CD investigations of Pre in EPA at $92 \mathrm{~K}$ in Leiden: ${ }^{14,15}$ Also there, ring closure to Dehy or Lumi was not detected, although these compounds would be easily identified by their UV and CD spectra with a vibrational structure. Hence thermalized Pre in cold EPA is $t Z c$-Pre.

It is satisfactory that $t Z c$-Pre was calculated to be the lowestenergy conformer in the gas phase, in particular in the OH-equatorial conformer, ${ }^{9,41}$ which is stabilized in polar solvents such as EPA (see ref. 11 and 15, for example). The calculated energy differences are small, however.

The fact that the thermal reaction Pre $\rightarrow c E c$-Tachy shows no detectable side product (as apparently all the other reported $Z-E$ isomerizations ${ }^{8,9}$ ) also means that hot ground state reactions are practically negligible in this case. (The error limits of the measurements ${ }^{8}$ would probably be consistent with a few percent of such side products.) Because the molecular sizes and photon energies are the same, this suggests that in Dehy ring opening they are also (nearly) negligible, not only of minor importance as concluded from the CD measurements. Note that the mechanism via a conical intersection does not require any rotamerization (Section 3).

It is also worth noting that the assignment "thermal Pre = $t Z c$-Pre" was derived without invoking any estimate on a contribution of a hot-ground-state reaction.

\section{Summary and outlook}

Whereas the investigation of Dehy ring opening to Pre and subsequent $Z-E$ isomerization to Tachy by transient absorption in Garching ${ }^{1}$ revealed only the main conformer of Tachy $(t E c)$, the fluorescence detection in Florida found two additional ones ( $c E t$ and $c E c$ ), with $t E c$ still being the main product. ${ }^{8}$ The Florida group concluded that there must have been also three conformers of Pre produced by ring opening and believe that they detected them. They suggested that the initially formed $c Z c$-Pre is converted for the most part by a reaction in the hot ground state to $t Z c$ and $c Z t$-Pre (or $c Z c$-Pre with opposite helicity ${ }^{9}$ ), which are then photoisomerized to the three Tachy conformers. If after this rotamerization in situ Pre were mainly $t Z c$-Pre, thermalized Pre must be $c Z c$-Pre, ${ }^{8}$ because the photochemical products are different for the two cases. The Florida group then ascribed DBT-type $Z-E$ isomerizations to all Pre conformers. When Lumi was used to prepare $\operatorname{Pre}^{9}$ (only $c Z c$-Pre was found), the authors believed to have seen a DBT accompanied by a (simultaneous?) axial-to-equatorial conformer rearrangement, in part followed 
by a change of helicity in the hot ground state of Tachy (Scheme 2b). The latter process was also induced photochemically (Section 3.1).

However, hot- $\mathrm{S}_{0}$ reactions are in these systems much less important than postulated, even at room temperature in solution (Section 4). This is clearly proven by time-resolved CD spectroscopy: upon ring opening of Dehy, the CD becomes (and stays until temperature equilibration and longer) more negative, as expected for $c Z c$-Pre as the product, whereas the other postulated Pre conformer in the claimed quantities would make the CD change positive (Section 4.2) within the first 5 ps after ring opening. If in situ Pre is mainly $c Z c$-Pre, thermal Pre must be a different conformer ( $t Z c$, Section 4.3). In fact, no trace of ring closure from thermal Pre was detected by fluorescence spectroscopy, ${ }^{8}$ although it should have been, if it were $c Z c$-Pre as claimed ${ }^{8}$ (Section 4.3). That is, the original assignments ${ }^{1}$ in situ Pre $=c Z c$-Pre (except possibly a few percent of rotamerized products) and cold thermal Pre $=t Z c$-Pre should be maintained (Scheme 2c). (There is no disagreement between ref. 1 and 8 on the Tachy conformers.) Hence also the original statement is maintained (or slightly modified): that upon photoisomerization, in situ Pre (from Dehy) and thermal Pre both follow an HT pathway or at least pass through an HT-type CI.

Section 4 also points out that Hula twist is concerted (Section 4.1). That is, it does not consist of two steps, a photochemical DBT and a subsequent SBR in the hot ground state (as suggested by Saltiel ${ }^{8}$ ).

The Florida group found on starting from Dehy not only the main Tachy conformer $(t E c)$ but two more, assigned in ref. 8 to $c E c$ and $c E t$-Tachy. A mechanism via an HT-type CI (Section 3.1) explains all these three Tachy conformers as products of the primary Pre form $(c Z c)$ and even predicts qualitatively the yield ratios and why $t E t$-Tachy is not formed. Even the observed site effect in the matrix can be understood. The molecular structure in the CI is that of a partially completed Hula twist (Section 3). The two main products result from HT- 6 and HT-7; the minor yield $\left(3 \%{ }^{8}\right.$ or $\left.20 \%{ }^{9}\right)$ from DBT can be understood as attempted HT (Section 3.1). The results from Florida ${ }^{8}$ can hence be considered a brilliant support for the HT CI mechanism. Nevertheless a DBT-type CI certainly exists also in this molecule. But obviously it is at higher energies and is not reached upon excitation in the near UV.

Section 3.2 again $^{1,30}$ points out that (in contrast to some opinions in the literature) HT is fully consistent with the NEER principle and conformer control. HT even offers additional possibilities of control, if the pretwist of $\mathrm{C}-\mathrm{C}$ single bonds is exploited. Examples are given. One example is also reported (the wavelength dependence of Pre photoisomerization), which is not pure conformer control: a sudden jump of the Tachy yield is also observed with a single Pre conformer $(c Z c)$ as the reactant. Obviously there is an internal energy threshold for this reaction. A model for such a barrier is presented in Fig. 3.

The slope down into the CI (which is about one third on the way towards a full HT) drives the molecule already without any volume constraint, although an external force can contribute (Section 3.1). A full HT in the absence of external forces was experimentally demonstrated e.g. with stilbene in the gas phase or in solution. ${ }^{31}$ An impressive example of free molecules showing all reactions expected from an $\mathrm{HT}$ CI is the recent work on substituted hemithioindigos in solution: ${ }^{40}$ both conformers of each, the $Z$ and $E$ isomer, could be isolated and individually investigated by NMR spectroscopy; they showed DBT, HT and SBR in various ratios. (Most recently, a similar system was used to demonstrate a photon-only driven molecular motor, exploiting SBR, DBT and $\mathrm{HT}^{72}$ ) These ratios and their various dependences can probably be interpreted by the pretwist model (Section 3.2). For unsubstituted hemithioindigo, excited state surfaces and their CIs with the ground state were calculated by the de Vivie-Riedle group. ${ }^{73}$ Several minima in the intersection space were found. Only one of the energetically accessible ones - an acrolein-type CI - is photochemically active (towards $Z-E$ isomerization); the others lead to only IC. An HT-type CI was found to lie higher than the Franck-Condon energy. It would be interesting to see, whether it is lowered by the pretwist, caused by substituents. From this work ${ }^{73}$ we can also learn that distortion along quite a number of coordinates can help bring $S_{1}$ and $S_{0}$ to an intersection (to a $\mathrm{CI}$ ). That is, one should be prepared to meet different types of CIs for $Z-E$ isomerization, although for Pre and other nonpolar polyenes an HT CI seems sufficient.

This quantum chemical work ${ }^{73}$ also emphasized the role of charge separation. So far, an HT CI has been thought to be a characteristic of nonpolar double-bond systems and the intersection of their covalent $2 \mathrm{~A}$ state with $\mathrm{S}_{0},{ }^{2}$ a rule which may need modification: in the substituted hemithioindigos the products point to an HT CI. Furthermore in Pre, HT was predicted to start also from the (ionic) 1B state by time-dependent DFT, ${ }^{41}$ a quantum chemical method not able to calculate the two-electron-excited $2 \mathrm{~A}$ state. Liu also pointed to possible HT in cyanine dyes ${ }^{23}$ (which are very polar), a view then also adopted by Rettig et al. ${ }^{74}$

Section 3.2 pointed out the importance of predistortion, be it by steric hindrance or by external forces. It can control the direction of motion long before the wave packet arrives in the branching region, that is, the CI; it also can select between different CIs. This concept is very successful in explaining many selectivities in photochemistry, such as the principle of least motion or the choice between different Woodward-Hoffmann allowed reactions; ${ }^{42}$ it is a generalization of conformer control. It can be interpreted by a (limited-time, Section 3.1) memory of the wave packet, from where it comes, or by a certain momentum conservation. While this seems intuitive, it deserves theoretical attention: the path of the wave packet is in general not straight, and it can enter the upper cone of the CI tangentially, so that the information on the original direction is lost.

It was shown in this contribution that an HT CI successfully explains the product spectrum of a relatively special system (although prototypical, or "considered the definitive evidence for $\mathrm{HT}^{, 8}$ ), previtamin $\mathrm{D}$, as observed in ref. 8. Of course, this CI is the basis of the many other observed cases of Hula twist, and the HT observations and the calculated molecular structure in the CI mutually support each other. But the HT CI explains also why the quantum yields for $Z-E$ and $E-Z$ isomerizations of polyenes usually do not add up to 1 (because the branching point - the CI - is not common for forward and backward reactions), which was not understood before. ${ }^{2}$ It was also used to rationalize why ring closure of dienes to cyclobutenes perfectly 
follows the Woodward-Hoffmann rules, whereas the corresponding ring opening is often unselective or even occurs selectively in the anti-Woodward-Hoffmann direction. ${ }^{42}$ The reason is that the $\mathrm{CI}$ for the (disrotatory) electrocyclic reaction is geometrically very close to the CI for (disrotatory) HT, so that a $Z-E$ isomerization channel can also be reached from the ring-opening path. The explanatory potential, demonstrated by these two examples, already justifies that the HT CI deserves attention.

\section{Conflicts of interest}

There are no conflicts to declare.

\section{Acknowledgements}

Open Access funding provided by the Max Planck Society.

\section{References}

1 A. M. Müller, S. Lochbrunner, W. E. Schmid and W. Fuß, Low-temperature photochemistry of previtamin D: a Hula-twist isomerization of a triene, Angew. Chem., Int. Ed., 1998, 37, 505-507.

2 D. Sampedro Ruiz, A. Cembran, M. Garavelli, M. Olivucci and W. Fuß, Structure of the conical intersections driving the cis-trans photoisomerization of conjugated molecules, Photochem. Photobiol., 2002, 76, 622-633.

3 P. Celani, M. Garavelli, S. Ottani, F. Bernardi, M. A. Robb and M. Olivucci, Molecular "trigger" for radiationless deactivation of photoexcited conjugated hydrocarbons, J. Am. Chem. Soc., 1995, 117, 11584-11585.

4 M. Olivucci, F. Bernardi, P. Celani, I. Ragazos and M. A. Robb, Excited-state cis-trans isomerization of cis-hexatriene. A CAS-SCF computational study, J. Am. Chem. Soc., 1994, 116, 1077-1085.

5 M. Garavelli, P. Celani, F. Bernardi, M. A. Robb and M. Olivucci, Force fields for "ultrafast" photochemistry: the $\mathrm{S}_{2}\left({ }^{1} \mathrm{~B}_{\mathrm{u}}\right)$ to $\mathrm{S}_{1}\left({ }^{2} \mathrm{~A}_{\mathrm{g}}\right)$ to $\mathrm{S}_{0}\left({ }^{1} \mathrm{~A}_{\mathrm{g}}\right)$ reaction path for all-trans hexa-1,3,5-triene, J. Am. Chem. Soc., 1997, 119, 11487-11494.

6 M. Garavelli, P. Celani, N. Yamamoto, F. Bernardi, M. A. Robb and M. Olivucci, The structure of the nonadiabatic photochemical trans-cis isomerization channel in all-trans octatetraene, J. Am. Chem. Soc., 1996, 118, 11656-11657.

7 M. Olivucci, M. A. Robb and F. Bernardi, in Conformational analysis of molecules in excited states, ed. J. Waluk, WileyVCH, New York, 2000, pp. 297-366.

8 C. Redwood, M. Bayda and J. Saltiel, Photoisomerization of pre- and provitamin D in EPA at $77 \mathrm{~K}$ : one-bond twist, not Hula-twist, J. Phys. Chem. Lett., 2013, 4, 716-721.

9 M. Bayda, C. E. Redwood, S. Gupta, O. Dmitrenko and J. Saltiel, Lumisterol to tachysterol photoisomerization in EPA glass at 77 K. A comparative study, J. Phys. Chem. A, 2017, 127, 2331-2342.

10 O. G. Dmitrenko, J. H. Frederick and W. Reischl, Ab initio study of conformational stability in previtamin D, vitamin D and related model compounds, J. Mol. Struct. THEOCHEM, 2000, 530, 85-96.
11 O. G. Dmitrenko, J. H. Frederick and W. Reischl, Previtamin D conformations and the wavelength-dependent photoconversions of previtamin D, J. Photochem. Photobiol., A, 2001, 139, 125-131.

12 O. G. Dmitrenko and W. Reischl, Molecular mechanics-based conformational analysis of previtamin D and its A-ring analogues, Monatsh. Chem., 1996, 127, 445-453.

13 P. A. Maessen, H. J. C. Jacobs, J. Cornelisse and E. Havinga, Photochemistry of previtamin D at $92 \mathrm{~K}$ : formation of an unstable tachysterol ${ }_{3}$ rotamer, Angew. Chem., Int. Ed., 1983, 22, 718-719.

14 P. A. Maessen, H. J. C. Jacobs, J. Cornelisse and E. Havinga, Photochemistry of previtamin D at $92 \mathrm{~K}$ : formation of an

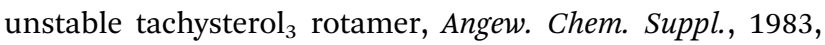
994-1004.

15 P. A. Maessen, The formation of toxisterols from previtamin D - Mechanistic studies, PhD work, Universiteit Leiden (NL), Leiden, 1983.

16 W. G. Dauben and D. J. H. Funhoff, Theoretical evaluation of the conformations of previtamin $\mathrm{D}_{3}$, J. Org. Chem., 1988, 53, 5070-5075.

17 E. Havinga, R. J. De Kock and M. P. Rappoldt, The photochemical interconversions of provitamin $\mathrm{D}$, lumisterol, previtamin D and tachysterol, Tetrahedron, 1960, 11, 276-284.

18 H. J. C. Jacobs and E. Havinga, Photochemistry of vitamin D and its isomers and of simple trienes, Adv. Photochem., 1979, 11, 305-373.

19 W. H. Laarhoven and H. J. C. Jacobs, in CRC Handbook of organic photochemistry and photobiology, ed. W. M. Horspool and P.-S. Song, CRC Press, Boca Raton, 1995, pp. 143-154.

20 R. S. H. Liu and G. S. Hammond, The case of mediumdependent dual mechanisms for photoisomerization: onebond-flip and Hula-twist, Proc. Natl. Acad. Sci. U. S. A., 2000, 97, 11153-11158.

21 R. S. H. Liu, Photoisomerization by Hula twist: a fundamental supramolecular photochemical reaction, Acc. Chem. Res., 2001, 34, 555-562.

22 R. S. H. Liu, in CRC Handbook of organic photochemistry and photobiology, ed. W. M. Horspool and F. Lenci, CRC Press, Boca Raton, 2004, p. 26.

23 R. S. H. Liu and G. S. Hammond, Examples of Hula twist in photochemical cis-trans isomerization, Chem. - Eur. J., 2001, 7, 4536-4544.

24 R. S. H. Liu, Hula-twist in photoisomerization: organic systems, Photogr. Sci. Photochem., 2002, 80, 81-87.

25 R. S. H. Liu and G. S. Hammond, Reflection on medium effects on photochemical reactivity, Acc. Chem. Res., 2005, 38, 396-403.

26 R. S. H. Liu, L. Y. Yang, Y. P. Zhao, A. Kawanabe and H. Kandori, in Supramolecular photochemistry: Controlling photochemical processes, ed. V. Ramamurthy and Y. Inoue, Wiley, Hoboken, 2011, pp. 593-605.

27 R. S. H. Liu, L. Y. Yang and Y. P. Zhao, in CRC Handbook of organic photochemistry and photobiology, ed. A. Griesbeck, M. Oelgemöller and F. Ghetti, CRC Press, Boca Raton, 2012, pp. 593-605. 
28 R. S. H. Liu and A. E. Asato, Photochemistry of polyenes. 22. The primary process of vision and the structure of bathorhodopsin - a mechanism of photoisomerization of polyenes, Proc. Natl. Acad. Sci. U. S. A., 1985, 82, 259-263.

29 R. S. H. Liu, D. Mead and A. E. Asato, Photochemistry of polyenes. 23. Application of the HT-n mechanism of photoisomerization to the photocycles of bacteriorhodopsin - a model study, J. Am. Chem. Soc., 1985, 107, 6609-6614.

30 W. Fuß, Hula-twist cis-trans isomerization: the role of internal forces and the origin of regioselectivity, J. Photochem. Photobiol., A, 2012, 237, 53-63.

31 W. Fuß, C. Kosmidis, W. E. Schmid and S. A. Trushin, The photochemical cis-trans isomerization of free stilbene molecules follows a Hula-twist pathway, Angew. Chem., Int. Ed., 2004, 43, 4178-4182.

$32 \mathrm{M}$. Klessinger and J. Michl, Excited states and photochemistry of organic molecules, VCH, New York, 1995.

33 F. Bernardi, M. Olivucci and M. A. Robb, Potential energy surface crossings in organic photochemistry, Chem. Soc. Rev., 1996, 25, 321-328.

34 W. Domcke, D. R. Yarkony and H. Köppel, Conical intersections, World Scientific, London, 2004.

35 B. S. Hudson, B. E. Kohler and K. Schulten, in Excited States, ed. E. C. Lim, Academic Press, New York, 1982, pp. 1-99.

36 W. Fuß, Y. Haas and S. Zilberg, Twin states and conical intersections in linear polyenes, Chem. Phys., 2000, 259, 273-295.

37 H. Petek, A. J. Bell, R. L. Christensen and K. Yoshihara, Fluorescence excitation spectra of the $\mathrm{S}_{1}$ states of isolated trienes, J. Chem. Phys., 1992, 96, 2412-2415.

38 W. Fuß, T. Schikarski, W. E. Schmid, S. Trushin, K. L. Kompa and P. Hering, Spectroscopy and ultrafast dynamics of the $2 \mathrm{~A}_{1}$ state of Z-hexatriene in gas phase, J. Chem. Phys., 1997, 106, 2205-2211.

39 B. G. Levine and T. J. Martínez, Ab initio multiple spawning dynamics of excited butadienes: role of charge transfer, J. Phys. Chem. A, 2009, 113, 12815-12824.

40 A. Gerwien, M. Schildhauer, S. Thumser, P. Mayer and H. Dube, Direct evidence for hula twist and single-bond rotation photoproducts, Nat. Commun., 2018, 9, 2510.

41 E. Tapavicza, A. Meyer and F. Furche, Unravelling the details of vitamin D photosynthesis by non-adiabatic molecular dynamics simulations, Phys. Chem. Chem. Phys., 2011, 12, 20986-20998.

42 W. Fuß, Predistortion amplified in the excited state, J. Photochem. Photobiol., A, 2015, 297, 45-57.

43 M. J. Bearpark, S. M. Larkin and T. Vreven, Searching for conical intersections of potential energy surfaces with the ONIOM method: application to previtamin D, J. Phys. Chem. A, 2008, 112, 7286-7295.

44 J. Liu, K. J. Boarman, N. L. Wendt and L. M. Cardenas, Regiospecific photoisomerization of fluorinated $(E, E)-1,4-$ diphenyl-1,3-butadienes, Tetrahedron Lett., 2005, 46, 4953-4956.

45 L. Y. Yang, R. S. H. Liu, K. J. Boarman, N. L. Wendt and J. Liu, New aspects of diphenylbutadiene photochemistry. Regiospecific Hula-twist photoisomerization, J. Am. Chem. Soc., 2005, 127, 2404-2405.
46 J. Saltiel, L. Cires and A. M. Turek, Conformer-specific photoconversion of 25-hydroxytachysterol to 25-hydroxyprevitamin $\mathrm{D}_{3}$ : role in the production of vitamin Ds, J. Am. Chem. Soc., 2003, 125, 2866-2867.

47 W. G. Dauben, B. Disanayaka, D. J. H. Funhoff, B. E. Kohler, D. E. Schilke and B. Zhou, Polyene $2{ }^{1} \mathrm{~A}_{\mathrm{g}}$ and $1^{1} \mathrm{~B}_{\mathrm{u}}$ states and the photochemistry of previtamin $\mathrm{D}_{3}$, J. Am. Chem. Soc., 1991, 113, 8367.

48 W. G. Dauben, B. Zhou and J. Y. L. Lam, Photochemistry of structural analogues of previtamin $\mathrm{D}_{3}$ : generality of the wavelength-dependent triene photocyclization, J. Org. Chem., 1997, 62, 9005-9008.

49 H. J. C. Jacobs, Photochemistry of conjugated trienes: Vitamin D revisited, Pure Appl. Chem., 1995, 67, 63-70.

50 J. Saltiel, L. Cires and A. M. Turek, in CRC Handbook of organic photochemistry and photobiology, ed. W. M. Horspool, and F. Lenci, CRC Press, Boca Raton, USA, 2004, ch. 27.

51 A. M. Müller, Tieftemperaturphotochemie von 7-Dehydrocholesterol und Previtamin D, Diplomarbeit, Technische Universität München, München, 1997.

52 W. Fuß and S. Lochbrunner, The wavelength dependence of the photochemistry of previtamin D, J. Photochem. Photobiol., A, 1997, 105, 159-164.

53 O. Dmitrenko and W. Reischl, Does Hula-twist contribute to $Z / E$ isomerization in solvents? Brief notes on the Hula-twist mechanism, Third Internet Photochemistry and Photobiology Conference, 2000.

54 H. J. C. Jacobs, J. W. J. Gielen and E. Havinga, Effects of wavelength and conformation on the photochemistry of vitamin D and related conjugated trienes, Tetrahedron Lett., 1981, 22, 4013-4016.

55 B. C. Arruda, J. Peng, B. Smith, K. G. Spears and R. J. Sension, Photochemical ring-opening and ground state relaxation in $\alpha$-terpinene with comparison to provitamin D3, J. Phys. Chem. $B, 2013,117,4696-4704$.

56 B. C. Arruda and R. J. Sension, Ultrafast polyene dynamics: the ring opening of 1,3-cyclohexadiene derivatives, Phys. Chem. Chem. Phys., 2014, 16, 4439-4455.

57 K. C. Tang, A. Rury, M. B. Orozco, J. Egendorf, K. G. Spears and R. J. Sension, Ultrafast electrocyclic ring opening of 7-dehydrocholesterol in solution: the influence of solvent on excited state dynamics, J. Chem. Phys., 2011, 134, 104503.

58 G. J. Collin, Photochemistry of simple olefins: chemistry of electronic excited states or hot ground state?, Adv. Photochem., 1988, 14, 135-175.

59 J. Benzler, S. Linkersdörfer and K. Luther, Density dependence of the collisional deactivation of highly vibrationally excited cycloheptatriene in compressed gases, supercritical fluids, and liquids, J. Chem. Phys., 1997, 106, 4992-5005.

60 U. Hold, T. Lenzer, K. Luther, K. Reihs and A. Symonds, Collisional energy transfer probabilities in the deactivation of highly excited aromatics, Ber. Bunsenges. Phys. Chem., 1997, 101, 552-565.

61 H. Hippler, K. Luther, J. Troe and H. J. Wendelken, Umimolecular processes in vibrationally highly excited cycloheptatrienes III: direct $k(E)$ measurements after laser excitation, J. Chem. Phys., 1983, 79, 239-246. 
62 S. Lochbrunner, W. Fuß, W. E. Schmid and K. L. Kompa, Electronic relaxation and ground state dynamics of 1,3cyclohexadiene and cis-hexatriene in ethanol, J. Phys. Chem. A, 1998, 102, 9334-9344.

63 S. Deb and P. M. Weber, The ultrafast pathway of photoninduced electrocyclic ring-opening reactions: the case of 1,3cyclohexadiene, Annu. Rev. Phys. Chem., 2011, 62, 19-39.

64 M. E. Squillacote, R. S. Sheridan, O. L. Chapman and F. A. L. Anet, Planar s-cis-1,3-butadiene, J. Am. Chem. Soc., 1979, 101, 3657-3659.

65 K. Nakanishi, Circular dichroism - principles and applications, Verlag Chemie, New York, 1994.

66 H. G. Wagner and W. Hug, Polarization and the sign of the long-wavelength Cotton-effects in chromophores of symmetry $\mathrm{C}_{2}$, Tetrahedron Lett., 1970, 11, 4765-4768.

67 W. Hug and G. Wagnière, The optical activity of chromophores of symmetry $\mathrm{C}_{2}$, Tetrahedron, 1972, 28, 1241-1248.

68 B. Matuszewski, A. W. Burgstahler and R. S. Givens, Preservation of chirality in the photochemical interconversion of trans- $\Delta 1,3$ hexalin and trans,cis,trans-cyclodeca-1,3,5-triene, J. Am. Chem. Soc., 1982, 104, 6874-6876.
69 J. Meyer-Ilse, D. Akimov and B. Dietzek, Ultrafast circulardichroism study of the ring-opening of 7-dehydrocholesterol, J. Phys. Chem. Lett., 2012, 3, 182-185.

70 N. A. Anderson, J. J. Shiang and R. J. Sension, Subpicosecond ring opening of 7-dehydrocholesterol studied by ultrafast spectroscopy, J. Phys. Chem. A, 1999, 103, 10730-10736.

71 W. Fuß, T. Höfer, P. Hering, K. L. Kompa, S. Lochbrunner, T. Schikarski and W. E. Schmid, Ring opening in the dehydrocholesterol previtamin D system studied by ultrafast spectroscopy, J. Phys. Chem., 1996, 100, 921-927.

72 A. Gerwien, P. Mayer and H. Dube, A photon-only molecular motor with reverse temperature-dependent efficiency, J. Am. Chem. Soc., 2018, 140, 16442-16445.

73 A. Nenov, T. Cordes, T. T. Herzog, W. Zinth and R. de VivieRiedle, Molecular driving forces for $Z / E$ isomerization mediated by heteroatoms: the example of hemithioindigo, J. Phys. Chem. A, 2010, 114, 13016-13030.

74 H. Wang, H. Zhang, W. Rettig, A. I. Tolmachev and M. Glasbeek, Femtosecond dynamics of the $S_{2}$ and $S_{1}$ fluorescence of ionic styryl dyes in polar solvents, Phys. Chem. Chem. Phys., 2004, 6, 3437-3446. 